\title{
Papel del refuerzo vertebral mediante cifoplastia en el tratamiento de las fracturas dorsolumbares de índole no osteoporótico. Revisión del tema y análisis de 40 casos
}

\author{
R. Arregui; J.V. Martínez-Quiñones; J. Aso-Escario y J. Aso-Vizan
}

Servicio de Neurocirugía. Hospital MAZ. Zaragoza.

Resumen

Objetivo. Presentamos una serie de pacientes con fracturas toraco-lumbares agudas en los que se ha practicado un refuerzo vertebral mediante cifoplastia, bien de forma aislada (cifoplastia percutánea) bien combinada con una fijación transpedicular posterior (cifoplastia abierta). Analizamos la posibilidad de ampliar el uso de la cifoplastia a las fracturas vertebrales de causa no osteoporótica, así como combinarla a los métodos tradicionales de fusión posterior.

Método. En nuestro servicio, entre los años 2003 y 2005, se trataron 138 pacientes afectos de una fractura aguda toraco-lumbar. En 87 casos se apreció una fractura a un nivel; en 34 a 2 niveles y, en los 17 restantes en más de dos. Un tratamiento conservador (reposo en cama, fisioterapia y movilización progresiva con corsé), fue realizado en 65 pacientes $(47 \%)$. Los 73 pacientes restantes fueron tratados quirúrgicamente, realizándose un refuerzo mediante cifoplastia percutánea en 25 casos $(18 \%)$, ó una cifoplastia asociada a fusión vertebral posterior en otros 15 (11\%). Diferentes modalidades de fijaciones atornilladas se realizaron en los 33 pacientes restantes (24\%). Este último grupo no ha sido objeto del presente estudio.

La estancia media hospitalaria de los pacientes tratados conservadoramente fue de 29 días. Siete de ellos (11\%) experimentaron una mala evolución, mostrando en las secuencias $\mathbf{T} 2$ del control de resonancia magnética una persistencia de hiperintensidad en el soma vertebral, sugerente de edema local.

Todos los pacientes que fueron tratados mediante cifoplastia aislada (percutánea) o combinada (abierta) mantenían una integridad neurológica; fueron clasificados en dos grupos:

- "Grupo a": Tratados mediante cifoplastia percutánea $(n=25)$. El índice medio sagital de este grupo fue de $11^{\circ}\left(6^{\circ}-15^{\circ}\right)$. En 9 pacientes, el acuñamiento vertebral superaba el $25 \%$. La estancia media hospitala-

Recibido: 18-01-08. Aceptado: 1-03-08 ria de este grupo fue de 14 días.

- "Grupo b": Tratados mediante cifoplastia combinada a fijación transpedicular $(n=15)$ : El índice medio sagital fue de $23^{\circ}\left(13^{\circ}-40^{\circ}\right)$. Todos los casos presentaron un acuñamiento vertebral superior al $25 \%$ junto a una alteración del muro vertebral posterior. La estancia media hospitalaria de este grupo fue de 35 días.

Resultados El resultado clínico de los 40 pacientes sometidos a cifoplastia fue medido en base al estado laboral, la restricción en las actividades físicas y el uso de analgesia. 36 pacientes regresaron a su trabajo previo con excepción de 4 casos del "Grupo b". En 11 casos se registró una restricción ligera de la actividad física. El seguimiento medio del "Grupo a" fue de 47 meses (10-72). La corrección cifótica media conseguida en ellos, fue de $5,3^{\circ}$. El seguimiento medio del "Grupo b" alcanzó los 26 meses (9-54). En este caso la corrección cifótica media conseguida fue de $10,3^{\circ}$.

Como complicaciones técnicas apreciamos 3 roturas de balones y cinco fugas intradiscales.

Conclusiones. La cifoplastia debe considerarse como una alternativa $y / 0$ un tratamiento complementario a otros procedimientos clásicos de fusión-estabilización espinal para las fracturas vertebrales de causa no osteoporótica. Por lo tanto, debe ser ofrecida como alternativa terapéutica, cuando esté indicada, a los pacientes afectos de una fractura vertebral. Como ventajas de combinar la cifoplastia y la fijación transpedicular se encuentran, por un lado, la reducción del número de niveles fusionados (instrumentaciones cortas) y, por otro, conseguir por vía posterior estabilizaciones-remodelaciones de 360 grados.

PALABRAS CLAVE: Vertebroplastia. Cifoplastia. Fracturas toraco-lumbares.

Vertebral reinforcement by means of kyphoplasty in the treatment of non-osteoporotic thoraco-lumbar fractures. Study of $\mathbf{4 0}$ cases and review of the literature 


\section{Summary}

Object. We present a series of patients with acute thoraco-lumbar fractures in whom we performed balloon vertebroplasty (kyphoplasty), either alone (percutaneous) or combined to posterior transpedicular fusion (open kyphoplasty). We emphasize the possibility of extending the use of kyphoplasty to non-osteoporotic vertebral fractures, and combining this method with traditional posterior fusion procedures.

Methods. Between 2003 and 2005, 138 patients suffering from thoraco-lumbar acute fractures, were treated in our Department. 87 corresponded to one vertebral level fractures; 34 to two levels, and the remaining 17 patients had more than two vertebrae affected. 65 patients $(47 \%)$ received conservative therapy (rest in bed, physiotherapy, and subsequent progressive mobilization with cast). The remaining ones ( 73 cases; $43 \%$ ) were treated invasively, performing balloon vertebroplasty alone $(n=25)$, or kyphoplasty associated to posterior fusion in 15 cases $(\mathbf{1 1 \%})$. Different kinds of screw posterior fusions were performed in the remaninig pacients $(n=33 ; 24 \%)$. The latter group was not included in the present study.

In the conservatively treated group (CTG), seven patients $(11 \%)$ had a bad outcome, showing a persistency of hyperintensity in MRI-T2 sequences of the vertebral body, suggesting local edema. Mean hospitalization rate was 29 days in CTG.

None of the 40 patients treated with kyphoplasty alone or combined with fusion showed abnormalities in neurological examination. They were classified in two groups:

- "Group a": Kyphoplasty alone (n=25). Mean of sagital index in this group was $11^{\circ}\left(\right.$ range: $\left.6^{\circ}-15^{\circ}\right)$. In 9 patients, vertebral body collapse exceeded $25 \%$. Mean hospitalization rate was 14 days.

- "Group b": Kyphoplasty and posterior fusion techniques $(n=15)$ : Mean sagital index was $23^{\circ}$ (range: $13^{\circ}-40^{\circ}$ ). All the patients presented with a vertebral body collapse superior to $25 \%$. All of them had posterior body wall involvement. This group was treated by surgery (decompression and fusion) and open vertebral body kyphoplasty. Mean hospitalization rate was 35 days.

Clinical results of these 40 patients were measured by means of work status, restriction of physical activities and analgesic drug intake. Except for four patients of "Group b", 36 returned to their work. In 11 cases a slight reduction of physical activity was registered.

Average "Group a" follow-up was 47 months (range: 10-72 months). A mean kyphosis correction of $5,3^{\circ}$ (sagital index) was reached in this group. Average
"Group b" follow-up was 26 months (range: 9-54). Mean kyphosis correction was $10,3^{\circ}$.

As for complications, we registered three balloon disruptions and five leakages into the disc.

Conclusions. Kyphoplasty could constitute an alternative and/or complementary treatment of traditional spinal stabilization-fusion procedures in non osteoporotic vertebral fractures. Therefore, it should be offered, when indicated, as a substantial possible part of the treatment, to the patients suffering from vertebral fractures. Additional advantages of combining kyphoplasty and posterior fusion are the possibility of reducing the number of fused levels (shorter instrumentations), and to perform a 360 degree stabilization-remodeling through a single posterior approach.

KEY WORDS: Ballon vertebroplasty. Kyphoplasty. Thoraco-lumbar fractures.

\section{Introducción}

Desde hace más de un siglo, el papel de la cirugía en el tratamiento de las fracturas del raquis ha sido controvertido $^{23,25,41}$. Mientras que las técnicas basadas en la reducción postural y el reposo se han mantenido prácticamente sin cambios, el tratamiento quirúrgico ha experimentado una constante renovación en los campos teórico y técnico $^{38,41,73,74}$.

Esta renovación ha derivado de dos elementos fundamentales. De un lado, el mejor conocimiento de la biomecánica y cinética de la columna vertebral, que nos ha permitido adaptar soluciones diferentes a los problemas clásicos de inestabilidad ${ }^{7}$. De otro, el gran avance en dispositivos de instrumentación, materiales y técnicas de refuerzo vertebral, que han puesto a nuestra disposición fantásticos métodos de reparación lesional, como es el caso de la cifoplastia con balón ${ }^{47}$.

Si consideramos la columna vertebral como una estructura en la que se hallan en equilibrio un juego de tensiones físicas que originan un conjunto íntegro y estable, la podríamos definir como un elemento con integridad tensional, del mismo modo que Kenneth Snelson consigue con su escultura denominada "torre de agujas"11,17. Este modelo de integridad tensional ya fue apuntado en el año 1977 por Whitesides $^{75}$.

Siguiéndolo, siempre que las fuerzas que se apliquen en los distintos brazos de palanca superen la resistencia vertebro-ligamentaria, se produciría una pérdida en la integridad del equilibrio de tensiones de la columna con un incremento gradual del fracaso espinal con el más que probable resultado de dolor, deformidad o daño neurológico ${ }^{43,54}$. $\mathrm{Si}$ además nos apoyamos en la teoría de las tres columnas ${ }^{21,45}$, podríamos considerar de forma genérica que una fractura 
confiere una deficiencia biomecánica (inestabilidad o pérdida de la tensegridad) cuando se afectan dos columnas contiguas (con afección tanto de elementos óseos como ligamentarios), incluso sin compromiso neurológico.

Por todos es sabido que una alteración de la columna posterior no es inestable de forma aguda a menos que se acompañe del fracaso del ligamento longitudinal posterior y del anillo fibroso posterior. En cambio cuando se altera la columna media, la fractura suele ser inestable. Aunque las alteraciones aisladas de la columna anterior son estables, existen algunas excepciones que la pueden transformar en inestables: a) fracturas por compresión en número de 3 ó más vértebras seguidas, b) una fractura por compresión única pero con una pérdida por encima del 50\% de la altura de la porción anterior del cuerpo vertebral, o con una angulación cifótica que supere los $20^{\circ}$ en un segmento $^{23,25,32,33,40,49}$.

La configuración intrínseca de la charnela toracolumbar contribuye de forma sustancial al tipo de lesión que puede sufrir. Así, a este nivel, concurren una serie de hechos que favorecerían la ubicación de fracturas: la falta de protección de las costillas, la falta de robustez de los cuerpos de las primeras vértebras lumbares y que el "momento de inclinación" intrínseco -derivado de la cifosis- no se vea contrarrestado ${ }^{41,57}$. En esta región serían más frecuentes las fracturas por flexo-compresión ${ }^{8,56}$.

Sin embargo, a la hora de clasificar los traumatismos toraco-lumbares, han existido muchas opiniones, tal y como podemos comprobar con la lectura de los trabajos de McAffe et al. ${ }^{50}$, Ferguson-Allen ${ }^{28}$, o Roy-Camille ${ }^{63}$. Nuestro grupo no pretende ahondar en esta desunión sino más bien conformar una estrategia terapéutica coherente. Para ello hemos tomado en consideración, entre otras, las clasificaciones propuestas por Panjabi/White ${ }^{58}$, Magerl et al. ${ }^{46}$, McCormack et al. ${ }^{52}$, o Chedid/Green ${ }^{14}$, y de su análisis hemos elaborado un esquema morfo-patológico en base al mecanismo de lesión, que hemos utilizado como guía terapéutica (Tabla 1).

Con el presente trabajo pretendemos aportar nuestra experiencia en el manejo de las fracturas dorso-lumbares de origen no osteoporótico sobre esta doble base, y enfatizar la utilidad del refuerzo vertebral mediante cifoplastia en el tratamiento integral de las mismas. Para ello repasaremos la biomecánica fracturaria, para presentar posteriormente nuestra casuística y resultados en el tratamiento de 138 casos de fracturas vertebrales tratadas por nosotros en tres años.

\section{Material y método}

\section{Casuística}

Durante el trienio 2003-2005 se ha asistido en el Servicio de Neurocirugía del Hospital MAZ un total de 138 pacientes con fracturas dorsolumbares, que se distribuyeron de la manera siguiente: 87 casos con afección a un nivel (51 de ellas a nivel de la charnela dorso-lumbar: 16 a nivel D12 y 35 a nivel L1), 34 casos a dos niveles y 17 casos de más de dos niveles. Por el mecanismo de producción los 138 pacientes fueron distribuidos de la siguiente forma: por flexo-compresión 73 casos, por carga axial (fundamentalmente precipitaciones) 52 casos, flexión/distracción 11 casos y por torsión 2 casos. La distribución por sexos fue: 102 varones y 36 hembras, con una edad media global de 41.3 años (18-64).

\section{Protocolo diagnóstico}

Incluyó una resonancia magnética $(\mathrm{RM})$ [sobre todo en la secuencia STIR -"short tau inversion recovery"- que muestra con claridad el edema, que aparece hiperintenso] y un escáner de las vértebras problema (útil para valorar retropulsiones del muro posterior en el conducto vertebral, así como para la realización de reconstrucciones tridimensionales) ${ }^{6,9,39,24,31}$. Buscamos establecer: (a) la integridad del ligamento longitudinal posterior (valorando la intrusión de elementos óseos en el conducto vertebral); (b) la deformidad espinal (definiendo el grado de acuñamiento -basándonos en el método de $\mathrm{Cobb}^{3}$ - (Tabla 2), y (c) la estabilidad primaria en base al índice sagital propuesto por Farcy et al: ángulo resultante de la intersección de las líneas rectas tangenciales al límite superior o inferior de la vértebra fracturada y al disco adyacente; al ángulo resultante (deformidad cifótica), hay que restarle un "factor de corrección" (o ángulo sagital basal a dicho nivel) de $+5^{\circ}$ para las vértebras D10 y D11 y de $-10^{\circ}$ para las vértebras L2 y L3 $\left(0^{\circ}\right.$ a nivel de la charnela dorsolumbar): [índice sagital $=$ deformidad cifótica - (factor de corrección) con su signo]. Según este índice, se considera que una fractura tiende a ser inestable si supera los $15^{\circ 26}$. Clasificamos las fracturas según el esquema que anteriormente hemos expuesto.

\section{Manejo}

En todos los pacientes se llevó a cabo un reconocimiento y tratamiento de los posibles daños asociados. El daño neurológico se evaluó en base a la escala ASIA/ IMSOP $^{2}$ y la clasificación de Frankel ${ }^{29}$. Se definieron cinco grados: A, B, C, D y E, considerando como "Grado A" al daño neurológico completo por debajo del nivel dañado, $y$ como "Grado E" la función motora y sensorial normal.

Con excepción de los casos de indicación quirúrgica absoluta (deterioro neurológico progresivo, fractura inestable con riesgo de alteración neurológica), se les ofreció a los pacientes el suficiente soporte informativo para que pudieran entender las distintas opciones terapéuticas:

1. Terapia conservadora. Consistió fundamentalmente en reducción postural y encamamiento durante al menos 3-4 semanas, analgesia, medidas antitrombó- 
Tabla 1

Clasificación operativa de las fracturas vertebrales

\section{Fracturas del cuerpo vertebral por compresión}

(Valorar el complejo ligamentario posterior: por lo general intacto)

A. Fracturas de los platillos vertebrales

No suele haber acuñamiento o este es mínimo

a. Centrales (comprometen la porción central del platillo). En pacientes con el disco intervertebral sano.

b. Periféricas (comprometen la concha cilíndrica cortical). Suelen producirse en pacientes con discos degenerados.

c. Combinadas: aquellas que producen fisuras transversas que se extienden a lo largo de todo el platillo vertebral

B. Fracturas acuñadas (acuñamiento por lo general debido a la compresión del hueso esponjoso). En su producción se combina una:

- Carga axial, que se aplicaría ventralmente al eje de rotación instantáneo (ERI)

- Flexión (momento de inclinación): anterior, antero-lateral o lateral. En dependencia del grado de osteoporosis o de la intensidad del agente traumático podemos apreciar un acuñamiento:

- anterior (o antero-lateral) simple (sólo se afecta la columna anterior)

- anterior con afección de las columnas anterior y media

- con afección de las tres columnas. Es posible la intrusión del muro posterior del cuerpo vertebral en el conducto vertebral.

C. Fracturas en estallido (conminuta). En su producción se combina una:

- Carga axial, que se aplicaría por lo general sobre el ERI.

- Choque a alta velocidad, aunque se ha conseguido reproducirlas mediante fuerzas de compresión a baja velocidad en modelos finitos ${ }^{(67)}$.

En estas fracturas valoramos:

- el grado de intrusión de los fragmentos en el conducto vertebral

- el grado de lesión de la columna posterior

- el daño neurológico: un 60\% de los pacientes padecen una afección neurológica, habitualmente incompleta ${ }^{(35)}$

- la estabilidad

D. Modalidad: En el año 1983, Lindahl et al. ${ }^{(44)}$ describieron un nuevo patrón de fracturas que consideraron como inestables, en pacientes que sufrían caídas verticales (fracturas por aplastamiento-escisión de Magerl et al. ${ }^{(46)}$ ). Consistían en: (1) una rotura del disco superior, (2) un aplastamiento de la mitad superior del cuerpo vertebral, (3) una fractura sagital de la mitad inferior del cuerpo vertebral, (4) una intrusión de fragmentos en el canal vertebral, y (5) una fractura de las láminas.

Este tipo de fractura se ve con frecuencia en el medio laboral.

\section{Fracturas por distracción y/o torsión de los elementos vertebrales anteriores y posteriores}

A. Fracturas por flexión/distracción

Por lo general se ve afectado el complejo ligamentario posterior, (que podría asociarse a subluxación, dislocación o fractura facetaria), o los elementos óseos posteriores (láminas, pedículos, istmo) ${ }^{(13)}$. Puede añadirse ventralmente una fractura acuñada (de mayor o menor intensidad) y dorsalmente una lesión de la musculatura paraespinal y del tejido celular subcutáneo.

A valorar el daño neurológico y el grado de inestabilidad.

\section{B. Fracturas por hiperextensión}

Raras. En dependencia de la importancia de las fuerzas que intervengan se pueden afectar las tres columnas. Su modalidad más grave, la dislocación posterior, se asocia a paraplejia completa.

\section{Fracturas por torsión (flexión-rotación)}

Las más devastadoras (afección tanto del raquis como de elementos neurales)

CT: obliteración del canal y signo del doble cuerpo vertebral; compresión/destrucción de la columna anterior 
Tabla 2

Clasificación de las fracturas por grado de acuñamiento

Grado 0: Situación normal.

Grado I: Acunamiento igual o menor al 25\% del soma vertebral, sin afección del muro posterior.

Grado II: Acuñamiento mayor del 25\% del soma vertebral, sin afección del muro posterior.

Grado III: Acuñamiento asociado a afección del muro posterior.

ticas, ortesis externa (de 2 a 3 meses) y, posteriormente rehabilitación.

2. Tratamiento quirúrgico. Este incluyó: bien la estabilización interna de la fractura o fracturas, bien el refuerzo vertebral mediante cifoplastia o, por último, la combinación de ambas.

En los casos tratados quirúrgicamente, se procedió de la siguiente manera:

1. Cuidados preoperatorios y consentimiento informado. Se procedió de forma estandarizada.

2. Técnica. No vamos a entrar en los distintos tratamientos quirúrgicos que se han realizado, puesto que no es la intención de este manuscrito, sino más bien en cómo se ha convertido el refuerzo vertebral mediante cifoplastia en un procedimiento a tener en cuenta en la resolución de algunas formas de fracturas toracolumbares. Así, en un principio nos limitamos a aplicar la técnica del refuerzo vertebral (cifoplastia percutánea) en las fracturas cerradas por compresión del cuerpo vertebral, con integridad del muro posterior vertebral, con un acuñamiento inferior al 50\% y $\sin$ riesgo de inestabilidad (indice sagital $<15^{\circ}$ ). Posteriormente aplicamos la cifoplastia en las fracturas por compresión con afección del muro posterior y, por último, en las fracturas "en estallido" del soma vertebral. En estas últimas, y en aquéllas con afección del muro y del arco posterior vertebral, e índice sagital $>15^{\circ}$, se combinó el refuerzo vertebral a una instrumentación transpedicular corta, cerrada en distracción, para así favorecer la corrección de los fragmentos desplazados. En caso necesario se completó con cirugía descompresiva.

La cifoplastia percutánea consiste en la introducción de un cemento óseo en el interior de un cuerpo vertebral colapsado. Sin embargo, previamente a la administración del cemento, a nivel de la vértebra fracturada, se inserta un globo o balón que se insufla con la finalidad de restaurar la altura del cuerpo vertebral y reducir la deformidad raquídea secundaria a la fractura. Al retirar el balón quedaría en el interior del cuerpo vertebral una cavidad o nido que permitiría introducir el material de refuerzo sin riesgo de extravasación ${ }^{47,48}$. Si bien el procedimiento técnico estándar es similar al que se realiza para el refuerzo de una vértebra fracturada osteoporótica, se dispone de utensilios (curetas) que permitirían remodelar las trabéculas óseas -en vértebras con hueso trabecular muy compacto- que favorecerían el insuflado de los balones.

3. Material de refuerzo. Se ha venido utilizando como biocemento diferentes formulaciones de fosfato tricálcico (KyphOs FSTMID o Calcibon ) o el polimetilmetacrilato (PMMA).

4. Control evolutivo. Todos los pacientes fueron controlados siguiendo el esquema propuesto por Herron y Turner en base al uso de analgésicos, la restricción de las actividades físicas y el regreso a la vida labo$\mathrm{ral}^{36}$. El tiempo mínimo de evolución ha sido de nueve meses.

5. Valoración del grado de restitución vertebral. Se realizó mediante morfometría vertebral sobre radiología simple y ficheros DICOM. Se agruparon los pacientes según el grado de acuñamiento vertebral, comparando su situación antes y después del procedimiento de refuerzo vertebral ${ }^{5}$.

\section{Resultados}

En nuestra serie, hemos apreciado un predominio del sexo masculino, probablemente debido a la mayor incidencia de accidentes laborales entre los varones.

De los 138 pacientes ingresados por fracturas dorsolumbares, en 65 se llevó a cabo un tratamiento conservador (pacientes afectos de fracturas trabeculares, de fracturas de platillo vertebral, o con un grado de acuñamiento de grados 0 ó I), y en cuarenta pacientes de los 73 restantes, aplicamos la técnica de refuerzo vertebral mediante cifoplastia (29\% del total -40/138-). Tabla 3.

PACIENTES TRATADOS CONSERVADORAMENTE. 65 casos (47\% del total). Todos ellos con un grado E funcional en el ASIA/IMSOP. Este grupo mantuvo una estancia hospitalaria media de 29 días, iniciándose una terapia rehabilitadora progresiva tras la misma. La retirada de la ortesis externa dependió del servicio de rehabilitación, así como su reincorporación laboral. En 7 pacientes (11\%; $7 /$ 65), se apreció una evolución inadecuada, que consistió en 
Tabla 3

Distribución de los pacientes

\begin{tabular}{|c|c|c|c|c|c|c|}
\hline \multirow[t]{2}{*}{ Tratamiento Realizado } & \multirow[t]{2}{*}{$\mathrm{N}^{\circ} \operatorname{casos}(\%)$} & \multicolumn{3}{|c|}{$\mathbf{N}^{\circ}$ niveles } & \multirow{2}{*}{$\begin{array}{l}\text { Sexo } \\
\mathrm{H} / \mathrm{M}\end{array}$} & \multirow{2}{*}{$\begin{array}{l}\text { Edad media } \\
\quad \text { (años) }\end{array}$} \\
\hline & & 1 & 2 & +2 & & \\
\hline Tratamiento conservador & $65 \quad(47)$ & 42 & 16 & 7 & 4718 & 39.8 \\
\hline $\begin{array}{c}\text { Tratamiento quirúrgico: } \\
\text { Cirugía convencional }\end{array}$ & $33 \quad(24)$ & 19 & 8 & 6 & 2310 & 42.3 \\
\hline Cifoplastia +/- cirugía estabilizadora & $40 \quad(29)$ & 26 & 10 & 4 & 328 & 40.6 \\
\hline TOTALES & $138(100)$ & 87 & 34 & 17 & 10236 & 41.3 \\
\hline
\end{tabular}

Tabla 4

Hallazgos radiológicos de los grupos "a" y "b"pre- y post-procedimiento

\begin{tabular}{|c|c|c|c|c|}
\hline \multirow{2}{*}{ Hallazgos radiológicos } & \multicolumn{2}{|c|}{ Grupo a } & \multicolumn{2}{c|}{ Grupo b } \\
\cline { 2 - 5 } & Pre-Q* & Post-Q & Pre-Q & Post-Q** \\
\hline Grado de acuñamiento: 0 & - & 3 pac. & - & 2 pac. \\
I & 15 pac. & 21 pac. & - & 9 pac. \\
II & 9 pac. & - & 4 pac. & 3 pac. \\
III & - & - & 11 pac. & - \\
\hline Índice sagital & $11^{\circ}\left(6^{\circ} \mathrm{a} 15^{\circ}\right)$ & $6^{\circ}\left(0^{\circ} \mathrm{a} 10^{\circ}\right)$ & $23^{\circ}\left(13^{\circ} \mathrm{a} 40^{\circ}\right)$ & $13^{\circ}\left(-2^{\circ} \mathrm{a} 18^{\circ}\right)$ \\
\hline
\end{tabular}

* Excluida la imposibilidad técnica: 1 caso

**Excluido el fracaso vertebral: 1 caso

la imposibilidad de reincorporación laboral por persistencia del dolor de carácter mecánico junto a espasmo muscular dorso-lumbar, que precisó del mantenimiento de la analgesia. En ellos se constató mediante un nuevo estudio de RM una persistencia de edema a nivel de la lesión fracturaria vertebral, o un agravamiento del acuñamiento vertebral. A todos ellos se les otorgó una incapacidad laboral para su trabajo habitual, aunque ninguno de ellos alcanzó una restricción severa de las actividades físicas según el esquema funcional de Herron y Turner.

PACIENTES TRATADOS QUIRURGICAMENTE CON CIFOPLASTIA. 40 casos (29\% del total). Todos presentaban una integridad neurológica, tanto motora como sensorial ("grado E" en el ASIA/IMSOP). De ellos, en 26 se apreció una fractura a un nivel, en 10 a dos niveles y en 4 a más de dos niveles. La charnela dorso-lumbar se vio afectada en 27 pacientes. La proporción de hombres/ mujeres fue de 4 a $1(32 / 8)$, con una edad media de 40.6 años (18 a 60). En 25 pacientes se realizó como procedimiento quirúrgico un refuerzo vertebral percutáneo (RVP) mediante cifoplastia ("Grupo a"). En otros 15 ("Grupo b") la cifoplastia fue aplicada a cielo abierto, en combinación con cirugía descompresiva y/o estabilizadora.

El tiempo medio del procedimiento de RVP en un paciente con un nivel afecto fue de 55 minutos (horquilla de 30 a 90). Cuando se combinó a cirugía abierta, el tiempo medio quirúrgico se incrementó por término medio en 40 minutos. El volumen medio de insuflación de los globos o balones fue de 2,5 cc (1-4) y el volumen medio de material de refuerzo administrado fue de 2,7 cc $(1,5-3,7)$. No fue preciso en ningún caso transfusión sanguínea.

En la Tabla 4 se puede apreciar los hallazgos radiológicos pre- y postquirúrgicos de los Grupos "a" y "b". Dentro del "Grupo a", 15 pacientes presentaron un acuñamiento por debajo del $25 \%$, mientras que los otros nueve lo superaron. En ningún caso se comprobó una afección del muro vertebral posterior. El índice sagital medio fue de $11^{\circ}\left(6^{\circ}-15^{\circ}\right)$. Tras el RVP mediante cifoplastia se consiguió un 'grado $0^{\prime}$ de acuñamiento en 3 pacientes y un 'grado $I^{\prime}$ en el resto. Se obtuvo una corrección cifótica media de 5,3 $3^{\circ}$. El tiempo medio de seguimiento de este grupo fue de 47 meses (10 - 72), y salvo en un paciente, tanto la corrección cifótica como el grado de acuñamiento se mantuvo estable (Figura 1).

La mayor parte de los pacientes incluidos en el "Grupo 


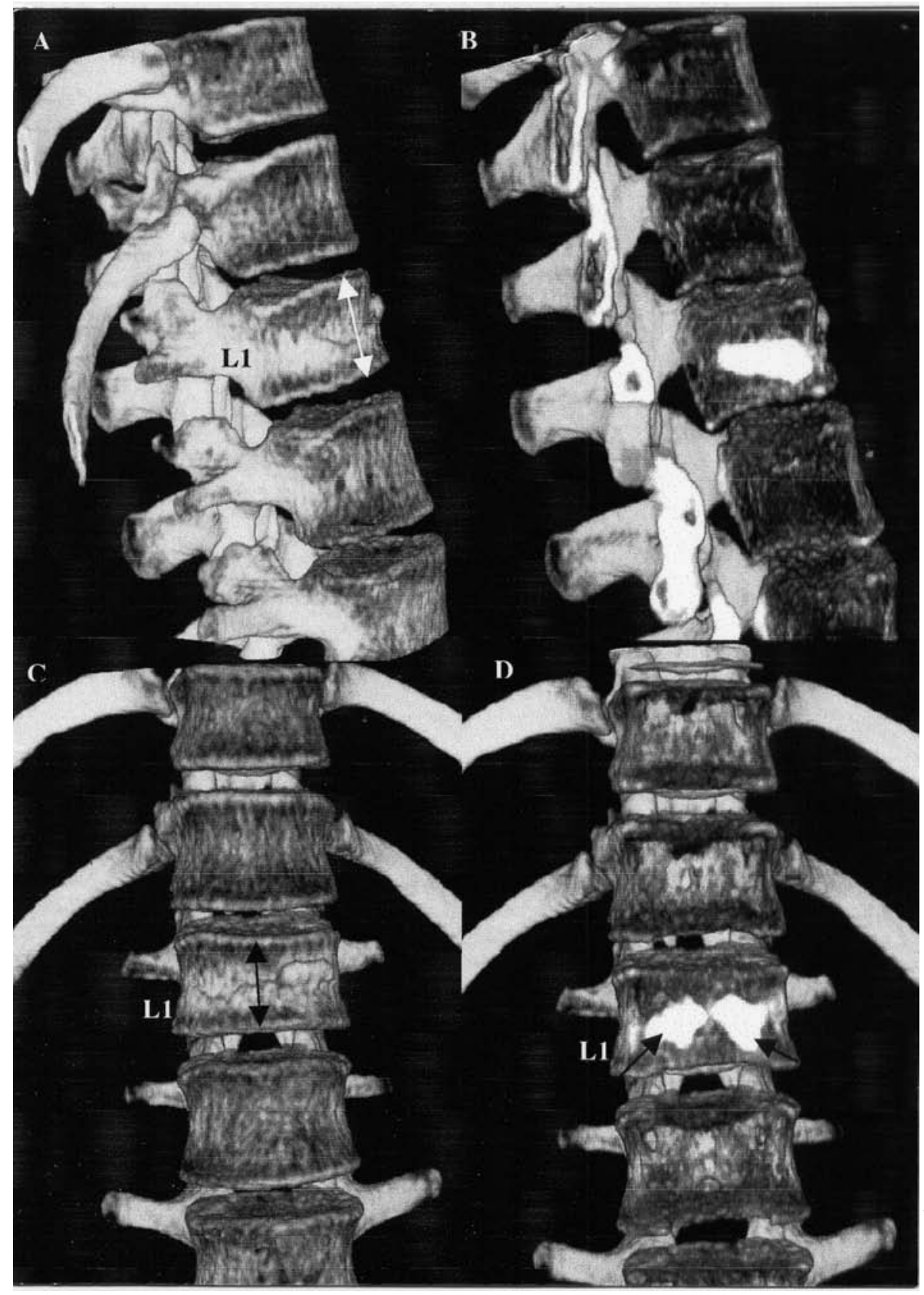

Figura 1. Varón de 25 años con fractura reparada de L1. Reconstrucciones $3 D$ de escáner toracolumbar en el que se puede apreciar el remodelado tras cifoplastia percutánea (A y C). En B y D puede apreciarse la distribución del cemento biológico intrasomático.

b" presentaban una afección del muro posterior, con un índice sagital medio de $23^{\circ}\left(13^{\circ}-40^{\circ}\right)$. Tras el procedimiento se consiguió un 'grado $0^{\prime}$ de acuñamiento en 2 pacientes, un 'grado $I^{\prime}$ en 9, y un 'grado $I I^{\prime}$ en 3. El tiempo medio de seguimiento fue de 26 meses ( 9 - 54), y si no tenemos en cuenta el caso del paciente que sufrió un fracaso del soma vertebral, la corrección cifótica media que obtuvimos fue de $10,3^{\circ}$, manteniéndose ésta prácticamente sin modificación durante el seguimiento evolutivo (Figuras 2 y 3).

En la Tabla 5 se puede apreciar los milímetros de recuperación media de altura tras el procedimiento quirúrgico practicado.
Las complicaciones apreciadas en estos 40 pacientes, las hemos dividido en técnicas y evolutivas. Entre las técnicas apreciamos 3 roturas de balones ("Grupo a") y cinco fugas intradiscales ("Grupo b"). En un caso, incluido en el "Grupo a", el procedimiento no pudo realizarse al no poderse canular los pedículos vertebrales; se trataba de una mujer que padecía una fractura acuñamiento del 25\% del soma de la vértebra D7. Fue tratada de forma conservadora y no sufrió otras complicaciones.

Las roturas de balones se apreciaron sobretodo en casos en los que se demoró el procedimiento técnico y en los que la remodelación vertebral resultó muy laboriosa. 


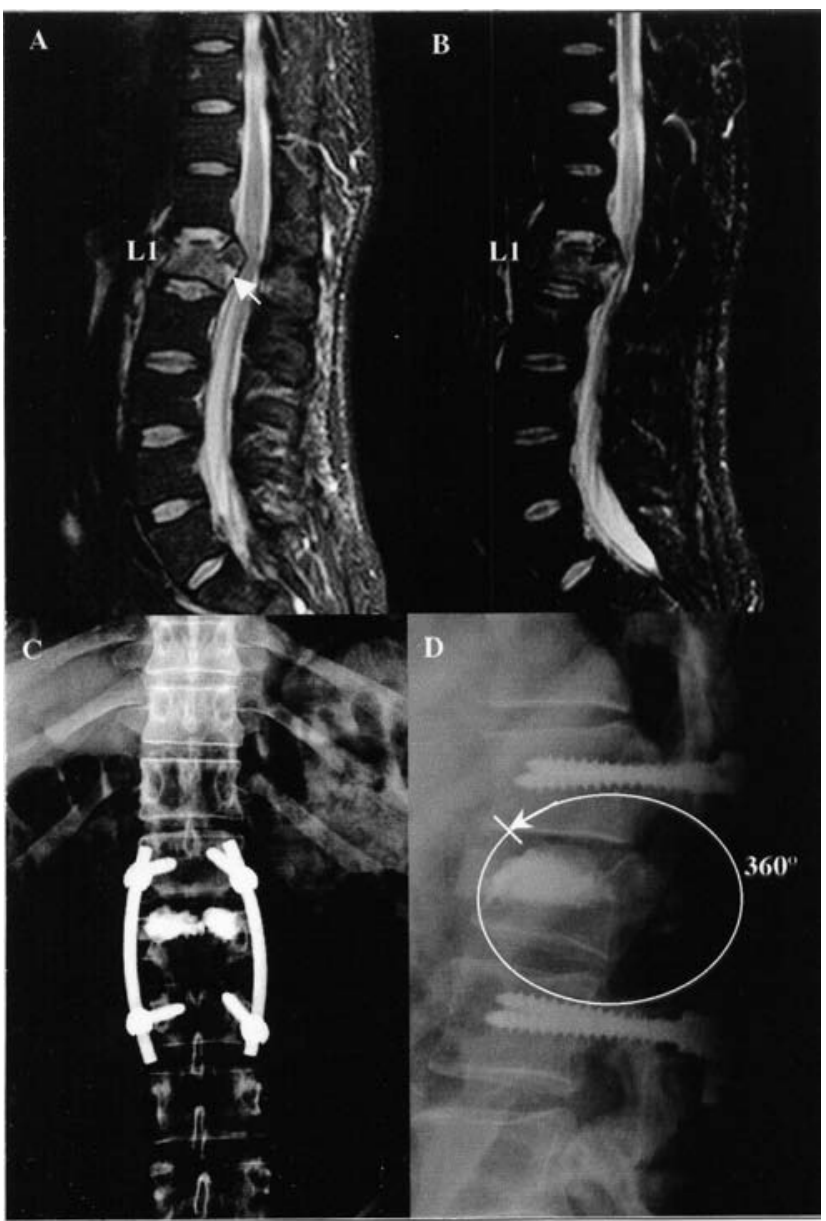

Figura 2. Varón de 33 años con fractura/estallido del soma de L1 tras precipitación. (A y B): diferentes secuencias de RM-T2 en la que se puede apreciar la afección del muro posterior (flecha negra), junto a la integridad del ligamento longitudinal posterior (flecha blanca). (C y D): Radiología simple en dos proyecciones a los seis meses de seguimiento; remodelación-estabilización 360 grados por vía posterior.

Tabla 5

Valoración del grado de restitución vertebral

\begin{tabular}{|l|c|c|}
\hline & \multicolumn{2}{|c|}{ Recuperación media de altura (mm.) } \\
\hline $\begin{array}{l}\text { Región } \\
\text { vertebral }\end{array}$ & $\begin{array}{c}\text { Grupo } \mathrm{a}^{*} \\
(23 \text { pacientes })\end{array}$ & $\begin{array}{c}\text { Grupo } \mathrm{b}^{\text {** }} \\
(14 \text { pacientes })\end{array}$ \\
\hline Posterior & 1,3 & 1,2 \\
\hline Media & 4,3 & 4,1 \\
\hline Anterior & 3,2 & 3,0 \\
\hline
\end{tabular}

* Excluidos la imposibilidad técnica y la pérdida $>2 \mathrm{~mm}$ de altura Post-Q. **Excluido el fracaso vertebral.

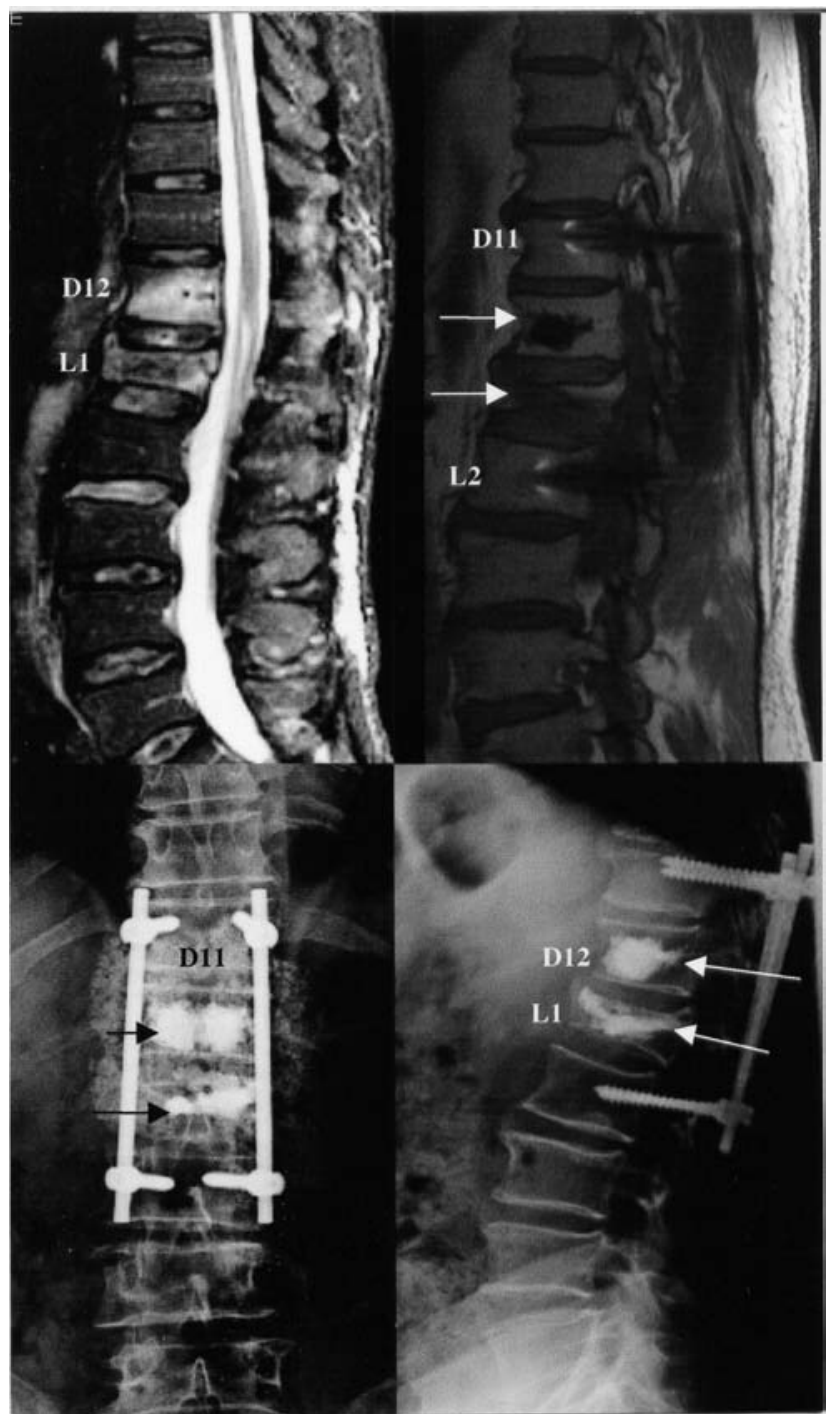

Figura 3. (A) Imagen de RM-T2 de varón de 58 años con fracturalestallido de L1 y fractura trabecular de D12 tras accidente laboral. (B) Imagen RM-T1 postratamiento quirúrgico por vía posterior: fijación transpedicular de D11 a L2 junto a refuerzo (cemento biológico) de D12 y $L 1$ (flechas blancas). (C y D) Estudio radiológico en dos proyecciones a los 5 meses de tratamiento en el que se puede apreciar el refuerzo vertebral (flechas) junto a la instrumentación vertebral de D11 a L2 practicada.

Las fugas intradiscales son relativamente frecuentes en las fracturas estallido, debido a que se asocian con frecuencia a fisuras/hendiduras que se extienden hasta los platillos vertebrales.

En cuanto a las complicaciones evolutivas apreciamos, dentro del "Grupo a", una pérdida parcial de la altura vertebral recuperada en un caso $(2,5 \mathrm{~mm}$. de pérdida, aunque sin repercusión clínica) y en el "Grupo b", un fracaso 
Tabla 6

Esquema funcional de seguimiento de Herron y Turner

\begin{tabular}{|c|c|c|c|}
\hline & puntaje & Grupo a (pac.) & Grupo b (pac.) \\
\hline Estado laboral & $\begin{array}{l}\text { Regreso al trabajo previo ............... (1) } \\
\text { Reubicación laboral ....................... (2) } \\
\text { Incapacitación laboral .................... (3) }\end{array}$ & $\begin{array}{l}23 \text { pac. } \\
2 \text { pac. } \\
-\end{array}$ & $\begin{array}{l}5 \text { pac. } \\
6 \text { pac. } \\
4 \text { pac. }\end{array}$ \\
\hline $\begin{array}{l}\text { Restricción } \\
\text { actividades físicas }\end{array}$ & 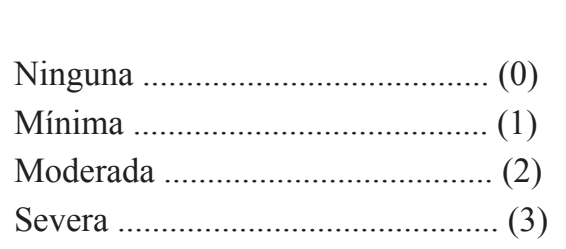 & $\begin{array}{l}20 \text { pac. } \\
5 \text { pac. } \\
- \\
-\end{array}$ & $\begin{array}{l}5 \text { pac. } \\
6 \text { pac. } \\
2 \text { pac. } \\
2 \text { pac. }\end{array}$ \\
\hline Uso analgesia & $\begin{array}{l}\text { Ninguna ........................................... (0) } \\
\text { Analgesia ocasional ........................ (1) } \\
\text { Analgesia habitual ......................... (2) } \\
\text { Toma ocasional de narcóticos .......... (3) } \\
\text { Toma habitual de narcóticos ........... (4) }\end{array}$ & $\begin{array}{l}21 \text { pac. } \\
4 \text { pac. } \\
- \\
- \\
-\end{array}$ & $\begin{array}{l}5 \text { pac. } \\
8 \text { pac. } \\
2 \text { pac. } \\
- \\
-\end{array}$ \\
\hline
\end{tabular}

vertebral en un varón de 45 años sometido a refuerzo vertebral asociado a laminectomía descompresiva sin fijación transpedicular.

Mediante el refuerzo vertebral buscamos además de un remodelado vertebral, un acortamiento de la estancia hospitalaria. Así, la estancia media del "Grupo a" fue de 14 días. Ahora bien, si tenemos en cuenta exclusivamente el tiempo medio de hospitalización tras el procedimiento, éste no superó las 48 horas. En el caso de los pacientes en los que se asoció cirugía estabilizadora y refuerzo vertebral, "Grupo b", la estancia media alcanzó los 35 días (21 - 45).

En base al esquema funcional de Herron y Turner, de los 40 pacientes, 36 se han reincorporado a la vida laboral activa, (la totalidad de los pacientes del "Grupo a"), mientras que cuatro $(10 \% ; 4 / 40)$ fueron catalogados como incapacitados para desarrollar su trabajo habitual. Once pacientes manifestaron una reducción mínima de sus actividades físicas. Veintiún pacientes del "Grupo a" y 5 pacientes del "Grupo b", no necesitaron de analgesia durante el control evolutivo (Tabla 6).

PACIENTES TRATADOS QUIRURGICAMENTE SIN CIFOPLASTIA. 33 casos ( $24 \%$ del total). 23 hombres y 10 mujeres, con una edad media de 42.3 años (21-62). En tres casos se comprobó un daño neurológico completo y en 15 una afección parcial de la función motora y/o sensorial. Este grupo no ha sido objeto de estudio en el presente manuscrito.

\section{Discusión}

No ha sido la intención de este manuscrito comparar el refuerzo vertebral mediante cifoplastia a otros procedimientos quirúrgicos utilizados en el tratamiento de las fracturas dorsolumbares de origen no osteoporótico, sino más bien estudiar las posibilidades de su uso en esta patología tan frecuente.

$\mathrm{Ni}$ decir tiene que la reducción postural y el encamamiento prolongado pueden conseguir la curación de un buen número de fracturas toraco-lumbares incluso con recuperación neurológica completas ${ }^{51,68}$, pero de todos es sabido que esta modalidad terapéutica implica una hospitalización mayor, un retraso en el inicio del tratamiento rehabilitador y puede incluso ocasionar morbilidad ${ }^{20,37,38,53,66}$. Knight et al resultan categóricos al establecer que el tratamiento conservador no se ve superado por el quirúrgico cuando la fractura vertebral a tratar presente un reducción de su altura en menos del $20 \%$, un ángulo de cifosis $<20^{\circ}$ y una reducción del conducto vertebral por debajo del $20 \%{ }^{41}$.

Una fractura acunada por debajo del $25 \%$ puede ser muy bien tolerada, sobre todo si se preserva la integridad del complejo ligamentario posterior y las estructuras óseas -fundamentalmente los pedículos- ${ }^{7}$, y el paciente afecto tras un tratamiento conservador puede llegar a realizar una vida cotidiana sin limitaciones ${ }^{23}$. Ahora bien, en el medio laboral, el paciente, aún en estos casos, puede experimentar una dorsalgia baja o lumbalgia alta irradiada en cinturón que le limite funcionalmente y que obligue, una vez alcanzado 
los plazos legales, a otorgarle una incapacidad laboral. Esta secuela dolorosa fue definida claramente por McCormack et al. ${ }^{52}$ por el "vacío" que en la columna anterior origina un acuñamiento vertebral. Además, existiría el riesgo de progresión de estas fracturas consideradas a priori como estables. En un $11 \%$ de los pacientes de nuestra serie tratados conservadoramente comprobamos este último aspecto. En este sentido una evaluación retrospectiva realizada por Ramieri et al. determinó que hasta el $60 \%$ de las fracturas toracolumbares sin daño neurológico asociado, tratadas de forma conservadora, que ellos estudiaron ( 28 del tipo Al y 32 del tipo A2 de la clasificación de Magerr ${ }^{46}$ ) progresaron en $5^{\circ}$ con el método de Cobb de medición de la cifosis, en relación a su estado previo ${ }^{62}$.

Chen et al. comunicaron el uso del RVP mediante vertebroplastia en un varón de 33 años que sufrió varias fracturas en estallido tras un accidente laboral, ante el fracaso del tratamiento médico conservador. Únicamente apreciaron una fuga intradiscal del material de refuerzo (polimetilmetacrilato) a nivel L4-L5, sin clínica asociada, reiniciando el paciente las actividades de la vida diaria a los tres meses del procedimiento ${ }^{15}$. Del mismo modo, Burton et al. ${ }^{10}$ establecieron la posibilidad del uso del refuerzo vertebral mediante vertebroplastia en las fracturas en estallido de origen osteoporótico, a raíz de la experiencia comunicada por Nakano et al..$^{55}$. Por otro lado, Gaitanis et al., han descrito el uso de la cementoplastia en "cascara de huevo" para reparar las fracturas en estallido de causa osteoporótica $^{30}$. Verlaan et al., sugirieron que el refuerzo vertebral mediante cifoplastia es un método seguro y factible para la reparación de las fracturas toracolumbares de origen traumático ${ }^{69}$.

Nuestro grupo viene utilizando desde el año 2003 el RVP mediante cifoplastia y sin osteosíntesis, para el tratamiento de las fracturas por compresión en flexión sin compromiso del muro posterior (tipo A de Magerl) con un excelente resultado ${ }^{4,48}$. Los pacientes del "Grupo a" así tratados, abandonaron los analgésicos en una media de 5 días; en un mes, por término medio, no presentaban limitaciones en las actividades cotidianas de la vida diaria $y$, fueron dados de alta laboral en un tiempo medio de tres meses $(2-3,5)$. Con respecto al análisis morfométrico de este grupo, comprobamos que si queríamos conseguir una remodelación adecuada del soma vertebral debíamos realizar de forma precoz el procedimiento. Cuanto más tiempo pasara entre la instauración de una fractura y el procedimiento de refuerzo vertebral, más dificultoso resultaría el remodelado del soma vertebral fracturado. El grado de recuperación media de la altura vertebral que así obtuvimos, se mantuvo prácticamente sin modificación durante el seguimiento de los pacientes (salvo en un caso), hecho que también ha sido reseñado por diferentes grupos con una recuperación de altura en la porción vertebral anterior de
3,7 $\mathrm{mm}$ a 4,3 $\mathrm{mm}$ y una recuperación de altura en la porción vertebral media de $4,3 \mathrm{~mm}$ a $4,7 \mathrm{~mm}^{30,60,72}$. No obstante, como Feltes et al, consideramos que el grado de recuperación de la altura vertebral no influye en el alivio del dolor ${ }^{27}$. Únicamente en el 13\% de los pacientes se consiguió un remodelado volumétrico compatible con la normalidad. En este sentido Verlaan et al., apreciaron, en un estudio realizado en un modelo cadavérico, que la corrección en la altura obtenida tras la insuflación del globo de cifoplastia en las vértebras fracturadas, se veía reducida en $2-3 \mathrm{~mm}$ cuando se retiraba el mismo, milímetros que no se recuperaban tras la introducción del cemento biológico ${ }^{70}$.

En otras situaciones (deformidad cifótica superior a $20^{\circ}$; compromiso del conducto vertebral superior al $50 \%$; etc.) resulta necesario implantar dispositivos espinales anteriores y/o posteriores, buscando conseguir por un lado, una adecuada estabilización espinal y, por otro, la mayor reducción posible de la deformidad ${ }^{19,26,32}$. Los dispositivos de implantación anterior son muy útiles para soportar fuerzas de carga axial, sin embargo están limitados para resistir fuerzas rotacionales y traslacionales. Es por ello que suelen acompañarse con sistemas de placas atornilladas. Para su uso debe establecerse la relación riesgo/beneficio. Se vienen utilizando en fracturas en estallido que afectan las columnas anterior y media con déficit neurológico, y en la intrusión del muro posterior en el conducto vertebral con reducción de al menos el 50\% del mismo y sin déficit neurológico ${ }^{34,40}$. Los dispositivos de implantación posterior deben cerrarse en distracción, y aunque ofrecen un menor riesgo técnico que los dispositivos anteriores, presentan un elevado porcentaje de fracasos a largo plazo (progresivas pérdida de la altura del cuerpo vertebral fracturado o angulación cifótica) $)^{12,18,42,64,76,77}$, por lo que se ha buscado el uso de instrumentaciones posteriores largas, a pesar de que ello implique una disminución del arco de movimiento raquídeo, o combinar ambos abordajes ${ }^{22}$, aunque se prolongue mucho el tiempo quirúrgico o el tratamiento resulte en ocasiones muy agresivo para un paciente con poca repercusión clínica postraumática. Weidenbaum y Farcy establecieron un algoritmo terapéutico en base al índice sagital y al número de columnas lesionadas en un segmento vertebral. Así indican un tratamiento conservador si el índice sagital es $<15^{\circ}$ y sólo se ve afecta una columna; un abordaje posterior si el índice sagital no supera los $25^{\circ}$ y se ven alteradas al menos dos columnas, y un abordaje combinado, anterior y posterior, si el índice sagital supera $\operatorname{los} 25^{\circ 73}$.

Como Sanderson et al. ${ }^{65}$, y Parker et al..$^{59}$, entre otros, en el caso de necesitar una osteosíntesis, fuimos partidarios de utilizar, siempre que pudimos, una fijación transpedicular (FTP) corta. Cho et al combinaron con éxito la FTP corta al refuerzo vertebral mediante vertebroplastia para el tratamiento de las fracturas toracolumbares en estallido, 
consiguiendo con ello tanto una corrección cifótica estable (corrección cifótica media de $6.4^{\circ}$ ), como un incremento mantenido de la altura vertebral anterior ${ }^{16}$. Conforme nuestro grupo fue avanzando en el conocimiento del refuerzo vertebral mediante cifoplastia como procedimiento remodelador de las columnas anterior y media, valoramos que su uso podría incluirse no sólo como una técnica a tener en cuenta para reparar cierto tipo de fracturas, sino que podría combinarse con otros procedimientos estándares del tratamiento fracturario toraco-lumbar, minimizando con ello el riesgo de complicación que podría derivarse del uso de una instrumentación corta, como así concluyeron Cho et $\mathrm{al}^{16}$. Al combinar ambas técnicas conseguimos: estabilizar la columna, descomprimir las estructuras neurales, dotar al cuerpo vertebral de un refuerzo mecánico y, remodelar su morfología. De este modo nos fue posible dotar de una "estabilidad circunferencial" (columnas anterior y posterior) a los pacientes en que combinamos ambos procedimientos. Es más, Verlaan et al., han comunicado, tras un estudio realizado en un modelo cadavérico, que el desplazamiento de los fragmentos óseos que se producen tras insuflar el globo de cifoplastia en las vértebras fracturadas en estallido, tanto a nivel dorsal como lumbar, es inferior al milímetro, no considerándolo de trascendencia clínica. Este desplazamiento fue independiente de la integridad o no del ligamento longitudinal posterior ${ }^{71}$.

Otros autores han comunicado su experiencia en la combinación de una osteosíntesis corta y un refuerzo vertebral ${ }^{1,16}$. Nuestro grupo, al igual que Cho et al., y Acosta et al., ha apreciado un grado de recuperación media del acuflamiento vertebral estable sin que se produzca una pérdida significativa de la corrección de la cifosis en el control evolutivo $^{1,16}$.

En la remodelación de una fractura vertebral aguda existen con frecuencias espículas óseas que pueden favorecer el pinchado del globo de insuflación. Las fugas intradiscales del material de refuerzo suelen producirse por rotura de los platillos vertebrales. En ocasiones pueden intuirse si el globo avanza de forma rápida (sin resistencia) hacia el platillo vertebral. No hemos apreciado patología asociada a las mismas en los pacientes que la han sufrido.

Con respecto al fracaso vertebral que apreciamos, comentar que el paciente padecía una fractura en estallido de L1, con afección del muro posterior vertebral pero con integridad del ligamento longitudinal posterior. Se realizó una laminectomía descompresiva sin osteosíntesis combinada a un refuerzo vertebral. El postoperatorio inmediato cursó sin incidencias, pero hacia al mes de la cirugía experimentó una sensación rara en la región intervenida, evidenciándose en el estudio radiológico simple el fracaso de la vértebra reforzada. De común acuerdo con el paciente se optó por una actitud expectante, reincorporándose a su vida laboral transcurridos 6 meses. El paciente se mantiene asintomático a día de hoy. Evidentemente, una laminectomía disminuye la estabilidad intrínseca del raquis vertebral, y más aún si el paciente sufre un fracaso de las tres columnas, como era el caso. La experiencia nos ha enseñado que deberíamos haber asociado en este caso una fijación posterolateral que confiriera estabilidad a la columna posterior. Es más, de no asociar al refuerzo vertebral en las fracturas en estallido una fijación transpedicular, desaconsejamos su uso ${ }^{61}$.

\section{Conclusiones}

1. La cifoplastia se presenta como un método útil y eficaz en el tratamiento de las fracturas no osteoporóticas.

2. Puede ser aplicado aisladamente o en combinación con los métodos de estabilización tradicionales.

3. Ningún otro procedimiento puede realizar, como la cifoplastia, un remodelado vertebral eficiente sin técnicas muy cruentas, reduciendo, así las secuelas derivadas de la deformidad vertebral residual.

4. Entre sus ventajas figuran la posibilidad de emplear instrumentaciones cortas y realizar una estabilización circunferencial (360 grados) por vía posterior.

5. En consecuencia, creemos debe constituirse como un tratamiento alternativo y/o complementario de otros procedimientos de estabilización espinal en fracturas vertebrales por compresión de origen no osteoporótico y, por lo tanto, ofrecerse al paciente como parte de las alternativas o complementos de su tratamiento.

\section{Bibliografía}

1. Acosta, F.L., Aryan, H.E., Taylor, W.R., Ames, Ch.P.: Kyphoplasty-Augmented Short-Segment Pedicle Screw Fixation of Traumatic Lumbar Burst Fractures: Initial Clinical Experience and Literature Review. Neurosurg Focus 2005; 18 (3). http: //www.medscape.com/viewarticle/501678.

2. American Spinal Injury Association, International Medical Society of Paraplegia. International Standards for neurologic and functional classification of spinal cord injury. Chicago, Ill: ASIA/IMSOP; 1992.

3. An, H.S., Balderston, R.A.: Juvenile kyphosis. In: The Spine, $3^{\text {rd }}$ ed. Rothman RH and Simeone FA eds. WB Saunders Co, Philapdelphia. 1992; pp: 485-499.

4. Arregui, C., Martínez Quiñones, J.V., Aso, J.: Fracturas dorsolumbares. Nuevas estrategias terapéuticas. Comunicación al IX Congreso Nacional de la SENC. Santander 19-22 de mayo de 2004.

5. Aso, J., Martínez Quiñones, J.V., Arregui, R.: Cifoplastia, fracturas dorso-lumbares y valoración de daño corporal. La repercusión de un nuevo tratamiento en los parámetros de 
estabilización y secuelas. Cuad Val 2006; 5: 33-39.

6. Baker, L.L., Goodman, S.B., Perkash, I., et al.: Benign versus pathologic compression fractures of vertebral bodies: assessment with conventional spin-echo, chemical-shift, and STIR MR imaging. Radiology. 1990; 174: 495-502.

7. Benzel, E.C.: Biomechanics of lumbar and lumbosacral spine fractures. In: Rea G, Miller C, eds. Spinal trauma: current evaluation and management. Neusosurgical topics. USA, AANS; 1993: 165-195.

8. Benzel, E.C., Larson, S.J.: Functional recovery after decompressive operation for thoracic and lumbar spine fractures. Neurosurgery 1986; 19: 772-778.

9. Blumenkopf, B.: Radiology and anatomy of lumbar and lumbosacral fractures. In: Rea G, Miller C, eds. Spinal trauma: current evaluation and management. Neusosurgical topics. USA, AANS; 1993: 157-163.

10. Burton, A.W., Rhines, L.D., Mendel, E.: Vertebroplasty and Kyphoplasty: a Comprehensive Review. Neurosurg Focus. 2005; 18 (3). http://www.medscape.com/viewarticle/ 501670.

11. Bustelo, J.A.: Equilibrio de tensiones. Tecnociencia 2. Abril 2006.

12. Carl, A.L., Tromanhauser, S.G., Roger, D.J.: Pedicle screw instrumentation for thoracolumbar burst fractures and fractures-dislocations. Spine 1992;17 (8 Suppl): S317-5324.

13. Chance, G.Z.: Note on a type of flexion fracture of the spine. Br J Radiol 1948; 21: 452.

14. Chedid, M.K., Green, C.: A review of the management of lumbar fractures with focus on surgical decision making and techniques. Contemp Neurosurg 1999; 21: 1-5.

15. Chen, J-F., Wu, Ch-T., Lee, S-T.: Percutaneous vertebroplasty for the treatment of burst fracturtes. Case report. J Neurosurg (Spine 1) 2004; 2: 228-231.

16. Cho, D.Y., Lee, W.Y., Sheu, P.C.: Treatment of thoracolumbar burst fractures with polymethyl methacrylate vertebroplasty and short-segment pedicle screw fixation. Neurosurgery 2003; 53: 1354-1361.

17. Ciencia y Nasa. Biología elemental. Extraños esqueletos: "Torre de agujas"; 19 jun 2002. http://ciencia.nasa.gov/ headlines/y2002/19/jun cytoskeletous.htm

18. Cusick, J.F., Yoganandan, N.: Algorithms for the treatment of lumbar and sacral fractures. In: Rea GL and Miller CA, eds. Spinal Trauma: Current evaluation and management. AANS: Neurosurgical Topics. USA, 1993: 197-206.

19. Danisa, O.A., Shaffrey, C.I., Jane, J.A., et al.: Surgical approaches for the correction of unstable thoracolumbar burst fractures: a retrospective analysis of treatment outcomes. J Neurosurg 1995; 83: 977-983.

20. Davies, W.E., Morris, J.H., Hill, V.: An analysis of conservative (non surgical) management of thoracolumbar fractures and fracture dislocations with neural damage. J Bone Joint Surg Am 1980; 62A: 1324-1328.

21. Denis, F.: The three column spine and its significance in the classification of acute thoracolumbar spinal injuries. Spine 1983; 8:817-831.

22. Dimar, J.R. II, Wilde, P.H., Glassman, S.D., et al.: Thoracolumbar burst fractures treated with combined anterior and posterior surgery. Am J Orthop 1996; 25: 159-165.

23. Domenicucci, M., Preite, R., Ramieri, A., et al.: Thoracolumbar fractures without neurosurgical involvement: surgical or conservative treatment? J Neurosurg Sci. 1996; 40: $1-10$.

24. Emery, S.E., Pathria, M.N., Wilber, R.G., et al.: Magnetic resonance imaging of the post traumatic spinal ligament injury. J Spinal Disord 1989; 2: 229-233.

25. Farcy, J.P., Weidenbaum, M.: A preliminary review of the use of cotrel-Dubousset instrumentation for spinal injuries. Bull Hosp Jt Dis Orthop Inst 1988; 48: 44-51.

26. Farcy, J.P., Weidenbaum, M., Glassman, S.: Sagittal index in management of thoracolumbar burst fractures. Spine 1990; 15: 958-965.

27. Feltes, C., Fountas, K.N., Machinis, Th., et al.: Immediate and Early Postoperative Pain Relief After Kyphoplasty Without Significant Restoration of Vertebral Body Height in Acute Osteoporotic Vertebral Fractures. Neurosurg Focus. 2005; 18 (3). http://www.medscape.com/viewarticle/501674.

28. Ferguson, R.L., Allen, B.L. Jr.: A mechanistic classification of thoracolumbar spine fractures. Clin Orthop 1984; 189: 77-88.

29. Frankel, H.L., Hancock, D.O., Hyslop, G., et al.: The value of postural reduction in the inicial management of closed injuries of the spine with paraplegia and tetraplegia. Paraplegia 1969; 7: 179-192.

30. Gaitanis, I.N., Hadjipavlou, A.G., Katonis, P.G., et al.: Balloon kyphoplasty for the treatment of pathological vertebral compressive fractures. Eur Spine J 2005; 14: 250-260.

31. Gehweiler, J.A. Jr, Osborne, R.L. Jr, Becker, R.F.: The radiology of vertebral trauma. Philadelphia, Pa: WB Saunders Co; 1980.

32. Gertzbein, S.D., Court-Brown, C.M., Jacobs, R.R., et al.: Decompression and circumferential stabilization of unstable spinal fractures. Spine 1988; 13: 892-895.

33. Greenberg, M.S.: Handbook of neurosurgery. Sixth edition. Thieme Medical Publishers, New York. 2006.

34. Haas, N., Blauth, M., Tscherne, H.: Anterior plating in thoracolumbar spine injuries. Indication, technique, and results. Spine 1991;16 (Supp13): S 100-S111.

35. Hashimoto, T., Kaneda, K., Albumi, K.: Relationship between traumatic spinal canal stenosis and neurologic deficits in thoracolumbar Bursa fractures. Spine 1988; 13: 12681272.

36. Herron, L.D., Turner, J.: Patient selection for lumbar laminectomy and discectomy with a revised objective rating system. Clin Orthopaedics Related Res 1985; 199: 145-152.

37. Hitchon, P.W., Torner, J.C., Haddad, S.F., et al.: Management options in thoracolumbar burst fractures. Surg Neurol 
1998; 49: 619-627.

38. Hollowell, J.P., Maiman, D.J.: Management of thoracic and thoracolumbar spine trauma. In: Rea G, Miller C, eds. Spinal trauma: current evaluation and management. Neusosurgical topics. USA, AANS; 1993: 127-156.

39. Juneau, P.A. III, De Vito, D.P., Blumenkopf, B.: Cauda equina avulsion resulting from seat-belt flexion-distraction injury: "Cigar sign" on MRI. MRI decisions 1992; 6: 31-32.

40. Kaneda, K., Taneichi, H., Abumi, K., et al.: Anterior decompression and stabilization with the Kaneda device for thoracolumbar burst fractures associated with neurological deficits. J Bone Joint Surg Am 1977; 79: 69-83.

41. Knight, R.Q., Stornelli, D.P., Chan, D.P., et al.: Comparison of operative versus nonoperative treatment of lumbar burst fracture. Clin Orthop Relat Res 1993; 293: 112-121.

42. Kuklo, T.R., Polly, D.W., Owens, B.D., et al.: Measurement of thoracic and lumbar fracture kyphosis: evaluation of intraobserver, interobserver, and technique variability. Spine 2001; 26: 61-66.

43. Kuner, E.H., Kuner, A., Schlickewei, W., Mullaji, A.B.: Ligamentotaxis with an internal spinal fixator for thoracolumbar fractures. J Bone Joint Surg (Br) 1994; 76-B:1 07-112.

44. Lindahl, S., Willen, J., Nordwall, A., Irstam, L.: The crush-cleavage fracture. A "new" thoracolumbar unstable fracture. Spine 1983; 8: 559-569.

45. Louis, R.: Spinal stability as defined by the threecolumn spine concept. Mat Clin 1985; 7: 33-42.

46. Magerl, F., Aebi, M., Gertzbein, S.D., et al.: A comprehensive classification of thoracic and lumbar injuries. Eur Spine J 1994; 3: 184-201.

47. Martínez Quiñones, J.V., Aso, J., Arregui, R.: Percutaneous vertebral augmentation: vertebroplasty and kyphoplasty: operative technique. Neurocirugia. 2005; 16: 427-440.

48, Martínez Quiñones, J.V., Aso, J., Arregui, R.: Tratamiento de las fracturas dorsolumbares agudas no osteoporóticas mediante cifoplastia. Tres años de experiencia. Comunicación al XX Congreso Nacional de la Sociedad GEER. Valladolid, 2 y 3 de Junio de 2006.

49, McAfee, P.C., Yuan, H.A., Lasda, N.A.: The unstable burst fracture. Spine 1982; 7: 365-373.

50. McAfee, P.C., Yuan, H.A., Fredrickson, B.E., et al.: The value of computed tomography in thoracolumbar fractures: an analysis of one hundred consecutive cases and a new classification. J Bone Joint Surg Am 1983; 65A: 461-473.

51. McEvoy, R.D., Bradford, D.S.: The management of burst fractures of the thoracic and lumbar spine. Experience in 53 patients. Spine 1985; 10: 631-637.

52. McCormack, Th., Karaikovic, E., Gaines, R.W.: The load sharing classification of spine fractures. Spine 1994; 15 : 1741-1744.

53. Mumford, J., Weinstein, J.N., Spratt, K.F., et al.: Thoracolumbar burst fractures. The clinical efficacy and outcome of nonoperative management. Spine 1993; 18: 955-970.
54. Myklebust, J.B., Pintar, F., Yoganandan, N., et al.: Tensile strengh of spinal ligaments. Spine 1988; 13: 526-531.

55. Nakano, M., Hirano, N., Matsuura, K., et al.: Percutaneous trans-pedicular vertebroplasty with calcium phosphate cement in thetreatment of osteoporotic vertebral compression and burst fractures. J Neurosurg Spine 2002; 97: 287-293.

56. Oxland, T.R., Lin, R.M., Panjabi, M.M.: Three-dimensional mechanical properties of the thoracolumbar junction. $\mathrm{J}$ Orthop Res. 1992; 10: 573-580.

57. Panjabi, M.M., White, A.A.: Physical properties and functional biomechanics of the spine. In: White AA, Panjabi MM, eds. Clinical biomechanics of the Spine, second editon. Philadelphia, Pa: JB Lippincott; 1990: 1-83.

58. Panjabi, M.M., White, A.A.: Biomechanics of thoracic and thoracolumbar spine fractures. In: Rea G, Miller C, eds. Spinal trauma: current evaluation and management. Neusosurgical topics. USA, AANS; 1993: 117-125.

59. Parker, J.W., Lane, J.R., Karaikovic, B.E., et al.: Successful short-segment instrumentation and fusion for thoracolumbar spine fractures: a consecutive 41/2-year series. Spine 2000; 25: 1157-1170.

60. Phillips, F.M., Ho, E., Campbell-Hupp, M., et al.: Early radiographic and clinical results of balloon kyphoplasty for the treatment of osteoporotic vertebral compression fractures. Spine. 2003; 28: 2260-2265.

61. Phillips, F.M., Pfeifer, B.A., Lieberman, I.H., et al.: Minimally invasive treatments of osteoporotic vertebral compression fractures: vertebroplasty and kyphoplasty. AAOS Instructional Course Lectures 2003; 52: 559-567.

62. Ramieri, A., Villani, C., Nocente, M., et al.: Vertebral instability in non-neurologic thoracolumbar fractures: the predictive value of methods of measurement. Chir Organi Mov 2000; 85: 121-127.

63. Roy-Camille, R., Saillant, G.: Les traumatismos du rachis sans complication neurologique. Int Orthop 1984; 8: 155-162.

64. Roy-Camille, R., Saillant, G., Mazel, C.: Plating of thoracic, thoracolumbar, and lumbar injuries with pedicle screw plates. Orthop Clin North Am 1986; 17: 147-159.

65. Sanderson, P.L., Fraser, R.D., Hall, D.J., et al.: Short segment fixation of thoracolumbar burst fractures without fusion. Eur Spine J 1999; 8: 495-500.

66. Shen, W.J., Liu, T.J., Shen, Y.S.: Nonoperative treatment versus posterior fixation for thoracolumbar junction burst fractures without neurologic deficit. Spine 2001; 26: 1038-1045.

67. Shirado, O., Kaneda, K., Tadano, S., et al.: Mechanism of thoracolumbar burst fracture: a biomechanical study with finite element model and cadaveric spines. Presented at annual internacional society of the study of lumbar spine meeting. Boston, Mass. June 13-17, 1990.

68. Weinstein, J.N., Collalto, P., Lehmann, T.R.: Thoracolumbar "burst" fractures treated conservatively: a long-term 
follow-up. Spine. 1988; 13: 33-38.

69. Verlaan, J.J., van Helden, W.H., Oner, F.C., et al.: Balloon vertebroplasty with calcium phosphate cement augmentation for direct restoration of traumatic thoracolumbar vertebral fractures. Spine 2002; 27: 543-548.

70. Verlaan, J.J., van de Kraats, E.B., Oner, F.C., et al.: The reduction of endplate fractures during balloon vertebroplasty: a detailed radiological analysis of the treatment of burst fractures using pedicle screws, balloon vertebroplasty, and calcium phosphate cement. Spine. 2005; 30: 1840-1845.

71. Verlaan, J.J., van de Kraats, E.B., Oner, F.C., et al.: Bone displacement and the role of longitudinal ligaments during balloon vertebroplasty in traumatic thoracolumbar fractures. Spine. 2005; 30: 1832-1839.

72. Watts, N.B., Harris, S.T., Genant, H.K.: Treatment of painful osteoporotic vertebral fractures with percutaneous vertebroplasty or kyphoplasty. Osteoporos Int. 2001; 12: 429-437.

73. Weidenbaum, M., Farcy, J.C.: Surgical management of thoracic and lumbar burst fractures. The textbook of spinal surgery. Second Edition. Ed by K. Bridwell and R.L. Dewald. pp: 1839-1880. Philadelphia: JB Lippincott 1997.

74. White, A.A., Panjabi, M.M.: Clinical Biomechanics of the spine. $2^{\text {nd }}$ ed. Philadelphia, PA: JB lippincott; 1990: 330-342.

75. Whitesides, T.E. Jr.: Traumatic kyphosis of the thoracolumbar spine. Clin Orthop 1977; 128: 78-92.

76. Whitecloud, T.S. III, Butler, J.C., Cohen, J.L. et al.: Complications with the variable spinal plating system. Spine 1989; 14: 472-476.

77. Yoganandan, N., Larson, S.J., Pintar, F., et al.: Biomechanics of lumbar pedicle/plate fixation in trauma. Neurosurgery 1990; 27: 873-881.

Arregui, R.; Martínez-Quiñones, J.V.; Aso-Escario, J,; Aso Vizan, J.: Papel del refuerzo vertebral mediante cifoplastia en el tratamiento de las fracturas dorsolumbares de índole no osteoporótico. Revisión del tema y análisis de 40 casos. Neurocirugía 2008; 19; 537-550.

Correspondencia postal: Dr Martínez Quiñones, J.V. C/ Maria de Oviedo 1; Portal $1^{\circ}, 7^{\circ}$-B. 50008-Zaragoza 